\title{
Research on Wideband Channel Measurement System Based on Software Defined Radio ${ }^{1}$
}

\author{
Jianwen Ding ${ }^{2}$ 1,2 , Dan $\mathrm{Fei}^{2}$, Ruisi $\mathrm{He}^{2}$, Zhangdui Zhong ${ }^{2}$ and $\mathrm{Bo} \mathrm{Ai}^{2}$ \\ ${ }^{1}$ National Research Center of Railway Safety Assessment Beijing Jiaotong \\ University, Beijing, 100044, P. R. China \\ ${ }^{2}$ State Key Laboratory of Rail Traffic Control and Safety, Beijing Jiaotong \\ University, Beijing, 100044, P. R. China
}

\begin{abstract}
Based on the analysis of the impact of wireless channel measurements to the railway dedicated mobile communication quality under the high-speed railway, this paper presents a narrow pulse wireless channel sounding approach based on the software-defined radio (SDR) technology with a measurement system developed. The measurement system could realize the dynamic analysis and display of measurement data in real time without an oscilloscope as auxiliary measure equipment. It overcomes the deficiency of rectangular pulse forming used in traditional analog narrow pulse sounder and improves the effective measurement bandwidth. Then both closed loop and open loop verifying measurement system are constructed to verify the measurement ability. And the results prove this channel measurement system is able to measure the behavior of radio wave propagation, and that is very useful for channel modeling of railway wideband mobile communication system
\end{abstract}

Keywords: channel sounder, Software Defined Radio

\section{Introduction}

With the rapid development of the high-speed railway (HSR) in China, dedicated wireless communication system plays an important role, which provides the platform for the data transmission of train control system, heavy haul train locomotive synchronization operation and control system, the shunting operation system and dispatching communication system. Since the dedicated wireless communication system is closely related to the safety of train operation, the fault and hidden safety problems will directly lead to train control data and dispatching communication interrupt, safety assessment of the dedicated wireless communication system is an essential part in the whole safety assessment of rail traffic system.

The HSR propagation channel which usually has severe fading has a significant impact on the design and performance analysis of wireless train control systems [1]-[6]. As the Long Term Evolution (LTE) system is gradually used for commercial purpose worldwide, there has been an increasing interest of the application of wideband wireless communications in railways [2]. The investigation of wireless channel is very important in the design of wireless communication system. As a basic tool for the study of wireless channel, wireless channel sounder is thus worth of deep study. Narrow pulse based wireless channel sounder is a traditional approach to measure wireless channel on time-domain. Its design principle directly inosculates with the definition of channel impulse response, and wireless channel's impulse response can be measured without extra mathematical treatment,

${ }^{1}$ Supported by the National Natural Science Foundation of China under Grants U1334202 and 61222105, the Key Project of Chinese Ministry of Education under Grant 313006, and the Project of State Key Laboratory of Rail Traffic Control and Safety under Grant RCS2014ZT11.

2 To whom correspondence should be addressed. E-mail: jwding@bjtu.edu.cn 
so it has been used widely. At present, most narrow pulse channel sounders are realized through pure analog method, and need to be equipped with a high-speed oscilloscope for signal acquisition which has a complex structure and costs a lot, but it is unable to achieve a real-time data analysis. In addition, rectangular radio-frequency pulses generated through controlling physical switch on and off by rectangular narrow pulses used in pure analog method have wide side lobes in frequency domain, and are detrimental to improving system's multipath delay resolution but to reduce the measurement accuracy. What's more, this propety will reduce the effective measurement bandwidth because of the bad frequency flatness. The narrow pulse channel measurement method based on SDR technology which is a new technology widely used in the field of radio frequency communication for prototype system development and algorithm verification is proposed in this paper. This method is based on a universal hardware platform, so that researchers could focus on algorithm and software design rather than the hardware design, which could significantly shorten system development cycle. In addition, the idea of modularization can greatly reduce system costs and strengthen system expandability and reusability. Furthermore, this method can also be used to design pulse shape by the use of digital signal processing (DSP), to make up for the shortage of rectangular pulses. The use of specific software (such as LabVIEW and MATLAB) can also realize the real-time analysis and display of data, which is of great significance to reducing the blindness of measurement and saving storage space [7].

\section{Theoretical Analyses}

In the land wireless communication system, the signals sent by transmitter usually cannot reach receiver directly, they may be refracted, reflected, scattered and diffracted by such obstacles as valleys, buildings and trees, which is usually called as the multipath effect of wireless channel. Multipath effect refers to the enhancement or offset of signal amplitude finally generated by the vector superposition of sending signals at receiver after going through different propagation paths with fading, delay and phase shift. Therefore, the wireless channel could be represented by the time-varying channel impulse response $h(t, \tau)$, as follow:

$$
h(t, \tau)=\sum_{i=1}^{L} \beta_{i}(t) e^{j \emptyset_{i}(t)} \delta\left[\tau-\tau_{i}(t)\right]
$$

where $\beta_{i}(t)$ and $\emptyset_{i}(t)$ represent the amplitude and phase of the $i^{t h}$ multipath signal at the $t^{\text {th }}$ moment, whose time delay is $\tau$. Therefore, the received signal $y(t)$ can be represented as the convolution of sending signal $x(t)$ and channel impulse response $h(t, \tau)$, expressed as:

$$
y(t)=x(t) \otimes h(t, \tau)
$$

Fig.1 illustrates a disperse tapped-delay-line model, which is built based on (2). The major purpose of channel measurement is to obtain $h$ and $\tau$.

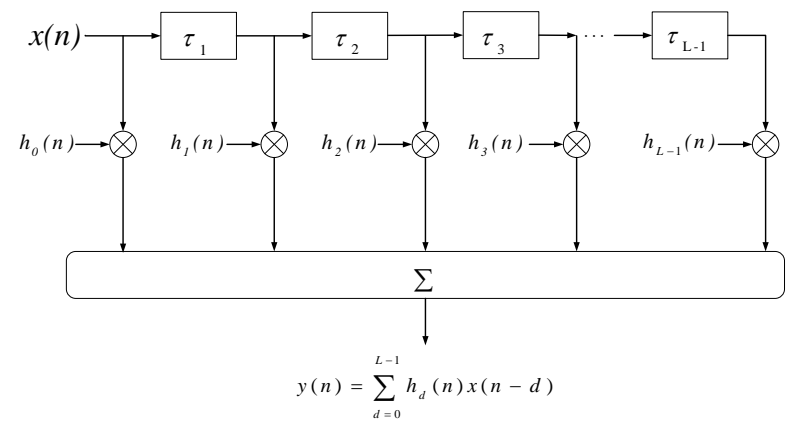

Figure 1. Disperse Tapped-Delay-Line Model 
The functional block diagram of the transmitter in the traditional pure analog-type narrow pulse wireless channel sounder is shown in Figure 2. It is mainly composed of narrow pulse generator, local oscillator, SPST switch, high-gain amplifier, pulse width modulator and control terminal [8]. Narrow pulse generator and local oscillator share a reference clock, and are respectively used to generate rectangular narrow pulses and continuous sine waves. Pulse width and local frequency can be regulated through pulse width modulator and control terminal. Pulse signal controls "on" and "off" of SPST switch from the output of local oscillator to the input of high-gain amplifier, as well as the switch-on time and pulse width. In this way, the output end of amplifier can generate the radio-frequency bursts whose duration is equal to rectangular pulse width. Furthermore, narrow pulse generator can simultaneously output a trigger signal which is synchronous with the rectangular pulse and can be used for the synchronous operation of transmitter and receiver.

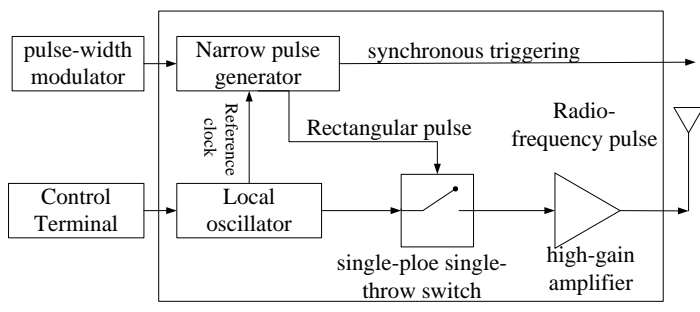

\section{Figure 2. Functional Block Diagram of the Transmitter in Narrow Pulse Channel Sounder}

The functional block diagram of the receiver is shown in Figure 3. It is mainly composed of low noise amplifier, local oscillator, mixer, band-pass filter, and log amplifier as well as such auxiliary equipment as control terminal and oscilloscope. RF input signals are sent to mixer to mix with local oscillator signals to be down-converted into intermediate frequency signals after amplified in low noise amplifier. Intermediate frequency signals are sent to log amplifier to receive envelope detection after the mirror component generated by frequency mixing are removed by band pass filter. The signals after envelope detection are low-frequency baseband analog signals and can be input into oscilloscope to complete the collection of data. An off-line analysis can be made on the collected data, so as to analyze channel characteristics and extract channel parameters.

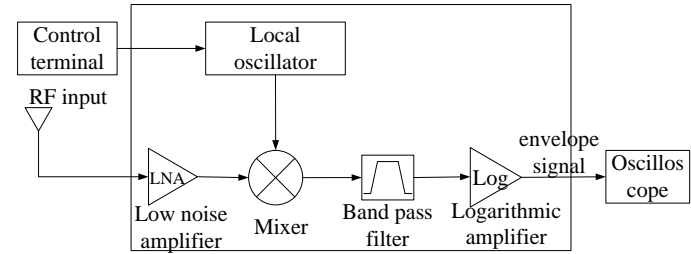
Figure 3. Functional Block Diagram of the Receiver in Narrow Pulse Channel
Sounder

\section{System Design}

The analysis in Chapter 2 shows that the transmitter of analog narrow pulse channel sounder generates radio-frequency pulse signals with SPST switch under the control of rectangular pulses and the receiver uses log amplifier for envelope detection after converting radio frequency pulse signals into intermediate frequency ones. Then, the signals are sent to the high-speed oscilloscope and saved. In SDR system, it can be approximated to the amplitude modulation and demodulation process of rectangular narrow 
pulses. Since demodulation process is done only in digital domain, this method can be directly used for data storage and analysis, whereas oscilloscope is no longer required for data acquisition in advance.

\subsection{System Structure and Principle}

The system structure of channel sounder proposed in this paper is illustrated in Figure 4. It is found that the whole system can be divided into two parts--software and hardware. The hardware has a common structure, while hardware configuration and control can be completed by software driver, therefore the key issue of the system design is the software part. The system can be functionally divided into transmitter and receiver. The transmitter is mainly composed of narrow pulse generator, amplitude modulation(AM) modulator, local oscillator, arbitrary waveform generator and up-converter. Narrow pulse generator is used to generate baseband narrow pulses whose bandwidth and repetition period are adjustable. AM modulator is used to generate two-way orthogonal signals of amplitude-modulated signals, whose modulation mode can be chosen as: double sideband amplitude modulation, single sideband amplitude modulation and vestigial sideband amplitude modulation. Rectangular pulse generator and AM modulator are realized by software. They are the key issue of system design, for which pulse pattern and amplitude modulation mode should be selected prudently. Local oscillator is a voltage-controlled oscillator (VCO) with fast tuning function, which is used to provide high-precision radio-frequency signal source. Arbitrary waveform generator is used for the digital-to-analog conversion of digital baseband signal and the low-pass filtering of analog baseband signal. The up-converter is applied to the up-conversion of analog baseband signals [9].

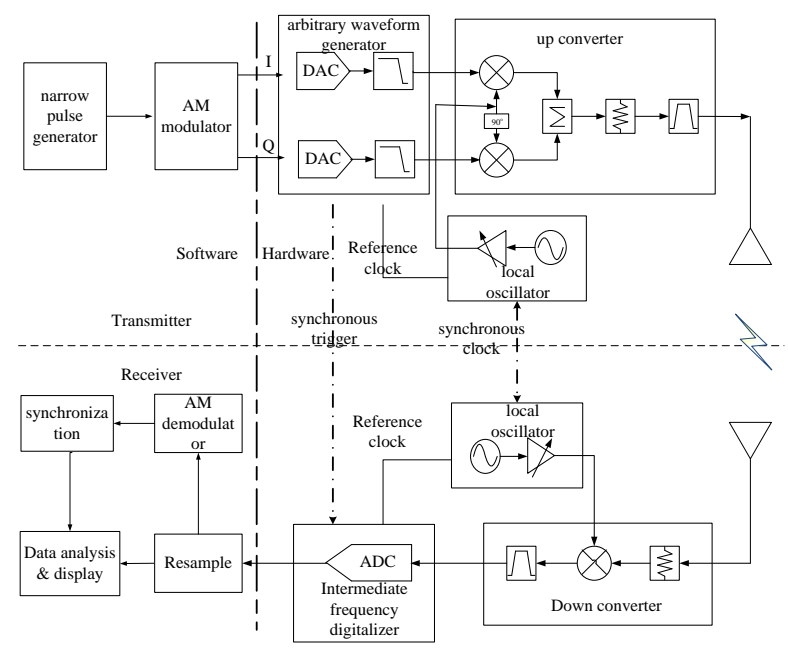

Figure 4. System Structure of Channel Sounder

The receiver is composed of local oscillator, down converter, intermediate-frequency digitalizer, AM demodulator, synchronization module and data analysis \& display module. Local oscillator is used to provide high-precision radio-frequency signal sources. Down converter, which is composed of low-noise pre-amplifier, mixer and band-pass filter, is used to accomplish down conversion of radio-frequency signals to the intermediate frequency whose center frequency is $187.5 \mathrm{MHz}$. Intermediate-frequency digitalizer is used for the analog-digital conversion and orthogonal demodulation of intermediate frequency signals. AM demodulator is used for the envelope detection of digital baseband signals and the restoration of rectangular pulse signals. Trigger capture module is used to set trigger level and capture triggering signals at the time of software synchronization. The module has the function of synchronizing transmitter and receiver when the line of sight (LOS) 
scenario is measured, and meanwhile, it is also the key to eliminating invalid data and carrying out average calculating operations after multiple measurements. Data analysis \& display module is used for the fundamental analysis and display of measured data.

To ensure the phase synchronization of signal reception \& transmission, the receiver and transmitter are connected to each other by a coaxial cable to share the reference clock in the receiver and transmitter. In addition, since the local oscillator shares reference clock with the intermediate-frequency digitalizer in the receiver and arbitrary waveform generator in the transmitter, this method can finish sampling synchronization between digital-analog converter (DAC) and analog-digital converter (ADC). To ensure that signal transmission in the transmitter is synchronous with signal acquisition in the receiver, the trigger pulses transmitted at the starting point of each transmitting cycle in the transmitter can be input into the intermediate-frequency digitalizer in the receiver for synchronous signal acquisition. Synchronization between the receiver and the transmitter is not only able to measure the absolute time delay of signal propagation, but also is an essential condition for the non-LOS (NLOS) scenario measurements.

\subsection{Software Design of Transmitter}

The software of transmitter mainly completes the generation of narrow pulse and AM modulation, in which the narrow pulse forming generation directly determine the RF pulse and is the key issue of the sounder design. In an analog narrow pulse channel sounder, radio-frequency pulses are generated by SPST switch on and off. That is, in this way, rectangular selection is infeasible for the shaping of radio-frequency pulse, and only fixed rectangular pulse shaping is available. No ideal waveform can be obtained easily even if a filter is added before radio-frequency signal output. The frequency spectrum of the radio-frequency narrow pulses generated through analog method is illustrated in Figure 5 which shows that spectrum energy is scattered in the whole signal bandwidth, roll-off is slow and side lobe occupies much energy. There is obvious depression between the mainlobe and sidelobe, which leads to bad frequency flatness, makes the emission power of frequency components of depression is low, leads to a low signal to noise ratio (SNR) of corresponding frequency components at receiver.

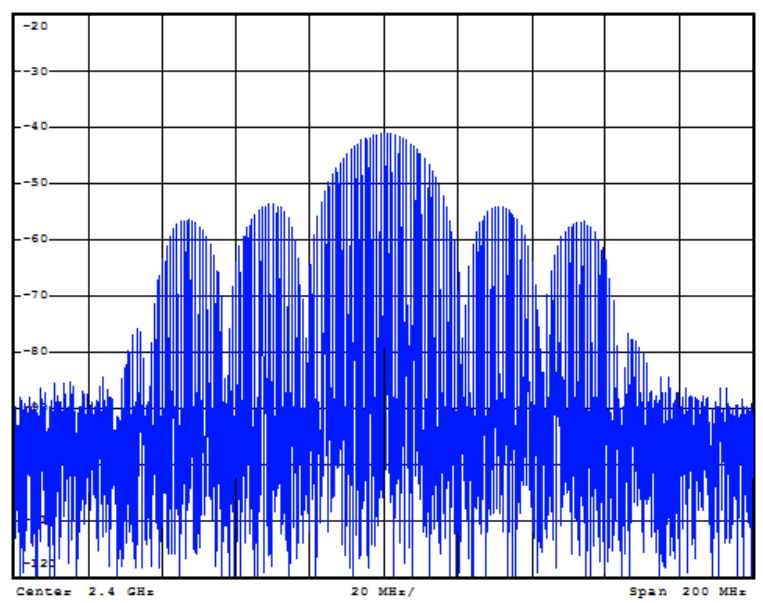

Figure 5. Frequency Spectrum of Rectangular Pulses

To solve the above problem, an arbitrary waveform shaping can be designed $\mathrm{s}$ to overcome the shortcomings of rectangular pulse shaping by using digital signal processing technology in SDR system. For this purpose, we choose Gaussian pulse as shaping waveforms. The Gaussian pulse is expressed as. 


$$
\begin{gathered}
y[n]=2 \pi a \sqrt{e} x_{n} f_{c} e^{-2\left(\pi x_{n} f_{c}\right)^{2}} \\
x_{n}=n * \Delta t-d, n=0,1, \ldots N-1
\end{gathered}
$$

where $a$ is amplitude, $d$ is time delay, $f_{c}$ is center frequency and $N$ is sample number.

The pulse signal frequency spectrum and time domain waveform after envelope detection obtained by the use of Gaussian pulse shaping are shown in Figure 6 and Figure 7. It is found that Gaussian pulse spectrum energy is concentrated, the roll-off is fast and the number of side-lobes is small. Time-domain signal envelope is less trailing, therefore, the shortcomings of rectangular pulse shaping can be overcome.

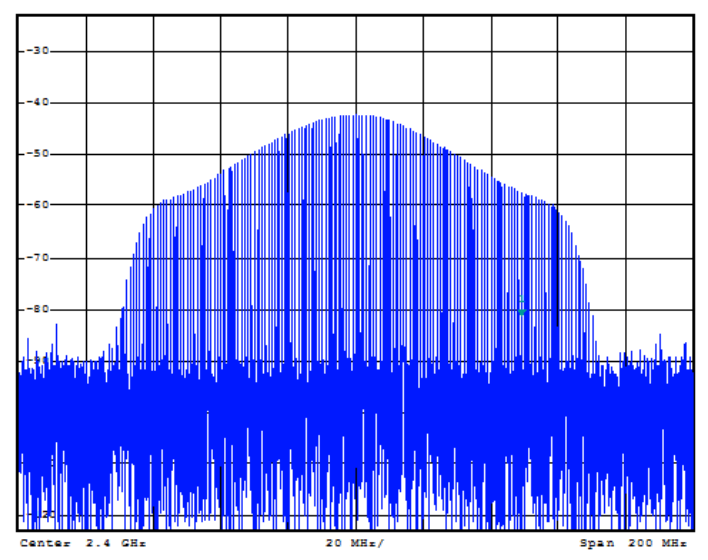

Figure 6. Signal Frequency Spectrum Generated by Gaussian Pulse Shaping

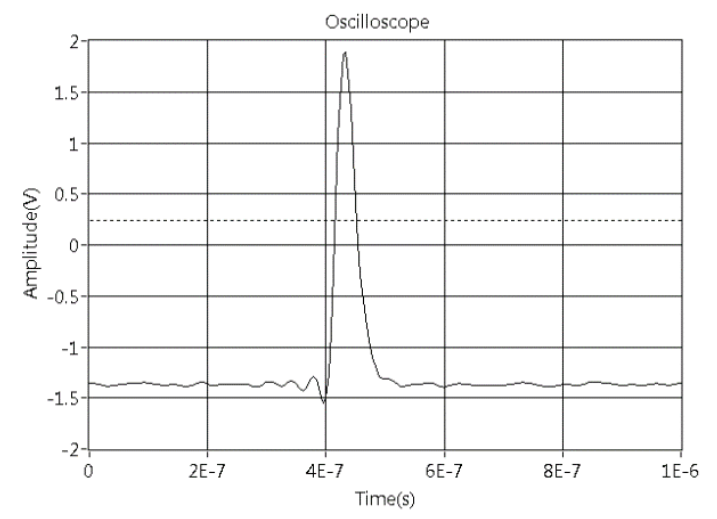

Figure 7. Signal Envelope Generated by Gaussian Pulse Shaping

\subsection{Software Design of Receiver}

Software design of the receiver mainly focuses on resample, AM demodulation, synchronization, data analysis and display. The design flow chart is showed in Figure 8. First, the baseband I/Q data are acquired from the onboard memory of intermediate-frequency digitalizer [10], and then the demodulated signal is sent to the synchronization module after finishing resampling and AM demodulation for triggering condition judgment. When the triggering condition is satisfied, the signal will be stored as useful signal; otherwise the signal will be abandoned. At last, accumulative total average calculation to stored useful signal is done and channel impulse response is extracted. 


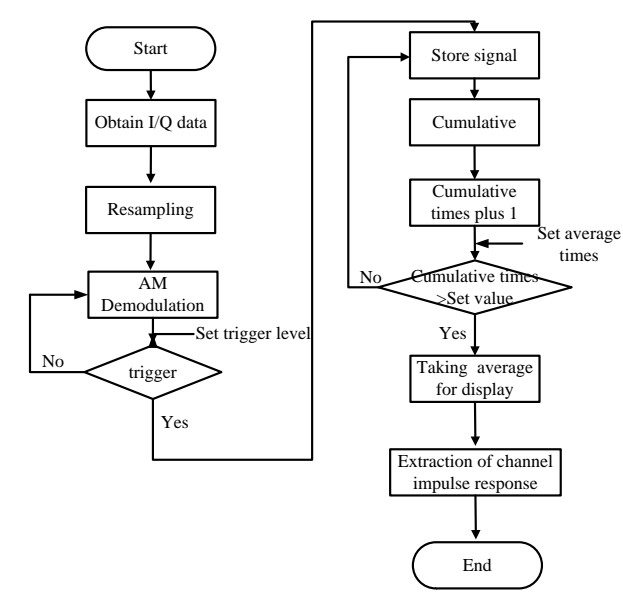

Figure 8. Treatment Scheme to Receiving End Software

Resample before AM demodulation can reduce the requirement of system to sample rate so as to ensure the range and accuracy of delay measurement while system working at extreme rate. Synchronization in software is realized by applying the method of level triggering, therefore the adjustment triggering level is the key factor, which determines the synchronization quality between receiver and transmitter and must be completed manually. It is not necessary to connect transmitter and receiver by coaxial cable for triggering synchronization, instead, a multipath signal, whose power is obviously stronger than other paths in receiving signals is necessary for trigger pulse. It is the reason that trigger synchronization is suitable for LOS scenario measurement, but hardware synchronization method in 2.1 is better for the NLOS scenario measurement.

The cumulative average of multiple measurements can be used to eliminate the influence of noise and burst interference to measurement in the condition of synchronization between transmitter and receiver, because only when the starting point of each pulse in the received signal is correctly aligned, i.e., synchronization is well completed between receiver and transmitter, and the average calculation is effective, otherwise, it will seriously affect accuracy of measurement. Since the software could process and analyze data on real time, the receiver can only store processing result, and does not need to store original data. It greatly saves storage space, and also reduces the requirement of data storage speed for dynamic measurement, which is important to wideband channel measurement.

\section{System Verification}

In order to verify the measurement function of the system, a closed loop and an open-loop measurement system are developed with a simulated channel and an actual channel as the measure objects in this paper. The simulated channel is generated by a dedicated radio channel emulator, and the actual channel is a corridor inside the main building of Beijing Jiaotong University.

The Structure of closed loop measurement system is showed in Figure 9. The transmitter uses SDR platform PXIe-5673E and the receiver uses SDR platform PXIe-5663E, which are all based on PXI bus technology [11] from U.S. National Instrument. This platform is designed with modularized structure and can be programmed flexibly with the LabVIEW. The system parameters of transmitter and receiver are showed in Table 1. 


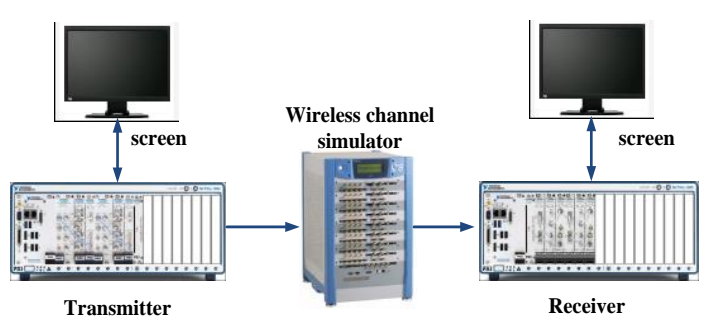

Figure 9. Structure of Closed Loop Measurement System

Table 1. System Parameter of Transmitter and Receiver

\begin{tabular}{|l|c|c|}
\hline & Transmitter & Receiver \\
\hline Frequency $(\mathrm{MHz})$ & $85 \sim 6600$ & $10 \sim 6600$ \\
\hline bandwidth $(\mathrm{MHz})$ & 100 & 50 \\
\hline $\begin{array}{l}\text { Maximum transmitting and receiving } \\
\text { power }(\mathrm{dBm})\end{array}$ & 10 & 30 \\
\hline Phase noise $(\mathrm{dBc} / \mathrm{Hz}, \quad 1 \mathrm{GHz})$ & 112 & 112 \\
\hline
\end{tabular}

EB Propsim C8 from Elektrobit Company of Finland [12] is used for channel simulation. It has 8 radio frequency channels of frequency domain from $350 \mathrm{MHz}$ to $3 \mathrm{GHz}$ and bandwidth of $75 \mathrm{MHz}$, and satisfies the requirement of standard model simulation for both GSM-R and LTE-R mobile communication systems.

The output signal from transmitter is sent to the input port of wireless channel simulation through RF cable and exported from output port of channel simulation instrument. Then the signal is sent to the receiver which measures channel impulse response. Channel parameter is shown in Figure 10. The amplitude and delay are set based on the COST207 Rural Area 6 taps model [13] and the amplitude fading distribution of each path is constant distribution, which is convenient for verifying the measurement accuracy of the system.

The measurement result is shown in Figure 11. It can be found that there are 6 taps in the measurement result. The relative amplitude between two adjacent taps is $4 \mathrm{~dB}$ and the relative amplitude between the strongest tap and the weakest tap is $20 \mathrm{~dB}$. The relative delay between two adjacent taps is $100 \mathrm{~ns}$ and the relative delay between the strongest tap and the weakest taps is $500 \mathrm{~ns}$. Therefore, the measurement result and the parameters of simulation are consistent.

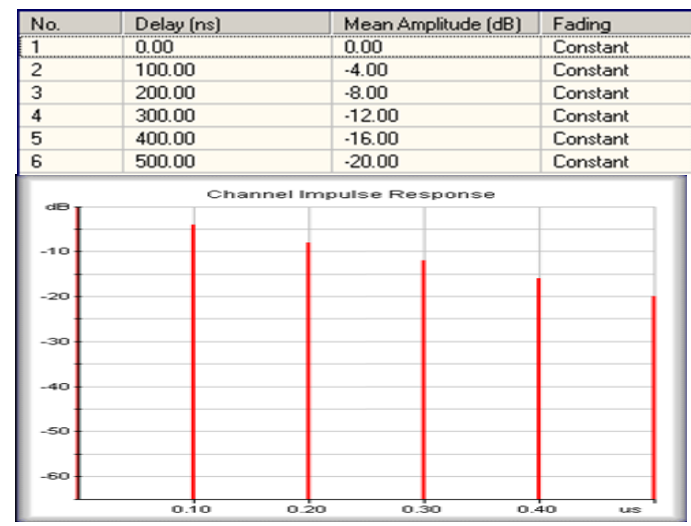

Figure 10. Model Parameter of Wireless Channel 


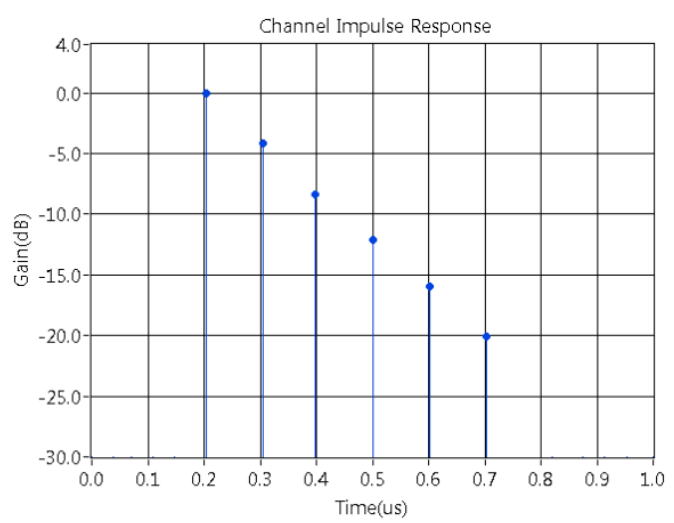

\section{Figure 11. Closed Loop Measured Result of Channel Impulse Response}

The open-loop validation scenario is shown in Figure 12. The whole corridor is about 50 meters, and the transmitter and receiver are located on the east side and central location of the corridor. By the west side, there are two doors, and the one close to the receiver is a metal door, the other one is a glass door. From our measurement results, it can be found that the two doors are the main scatterers, so they are marketed as Scatterer 1 and Scatterer 2 in Figure 12. The distance relationships of the main components measured with tape are shown in Figure 13.

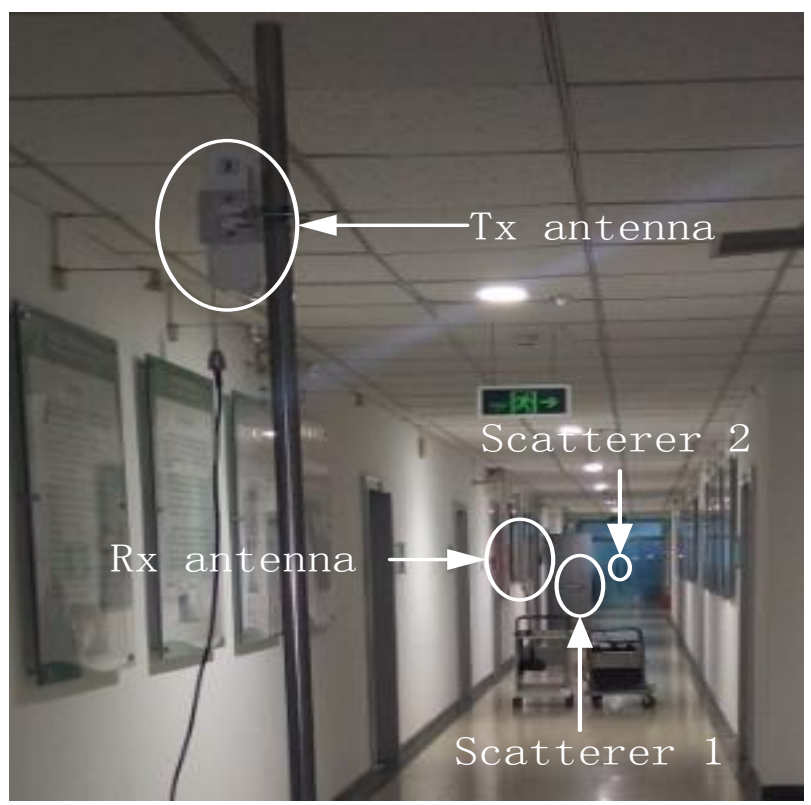

Figure 12. Schematic Diagram of Open-Loop Measurement Scenario

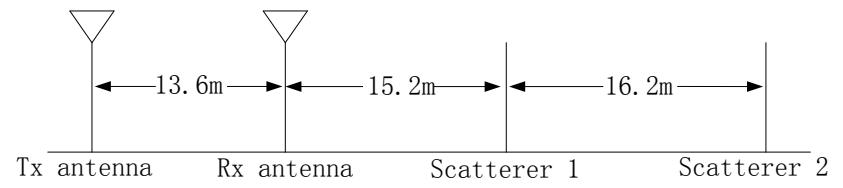

Figure 13. Distance Relationship of the Main Elements

The measurement result is shown in Figure 14, in addition to the LOS signal, there are two multipath signals which are the reflection signals generated by the two Scatterers. The relative amplitude to the first path are $-16.45 \mathrm{dBm}$ and $-18.76 \mathrm{dBm}$, and the relative delay are $90 \mathrm{~ns}$ and 205ns. According to the positional relationship in Figure 13. The theoretical delays of the 
reflected signal generated by Scatterer 1 is $\mathrm{s} * 2 / \mathrm{c}=89.3 \mathrm{~ns}$, where $\mathrm{s}$ is the distance from the scattering 1 to the receiving antenna. Similarly, the theoretical delay of the reflected signal generated by Scatterer 2 is $204 \mathrm{~ns}$. Considering the situation of the distance measurement device accuracy is not high, it can be found that the theoretical and measured delay are consistent.

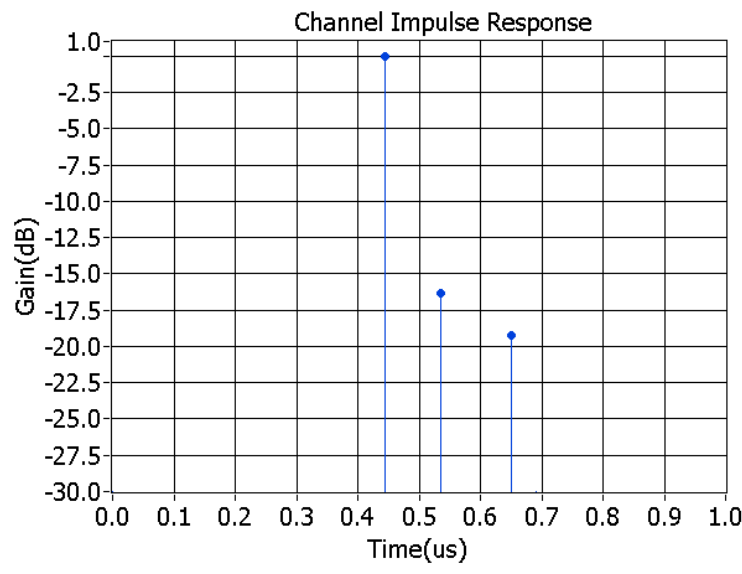

\section{Figure 14. Open Loop Measured Result of Channel Impulse Response}

\section{Conclusion}

In this paper, the narrow pulse wireless channel sounder based on the SDR technology is proposed, which has flexible and controllable structure, greatly saves developing period and cost, and overcomes the deficiency of rectangular pulse forming for traditional analog method. The measurement system does not need high speed oscilloscope as auxiliary equipment of measurement. This is because the analysis and dynamic display of measurement data can be finished in real time, and only the analysis result need to be stored, so that the requirement of system data storing speed and capacity are easy to meet. This system also has the function of dynamically displaying the analyzing result, therefore it can significantly reduce the blindness of measurement.. At the end of the paper, both closed loop and open loop verifying measurement system are constructed in this paper and the measurement results are consistent to the simulated or actual scenario. The measurement system will be upgrade from bandwidth and frequency range, so as to be used for channel measurement in $4 \mathrm{G}$ and $5 \mathrm{G}$ typical scenario in the future.

\section{References}

[1] R. He, B. Ai and Z. Zhong, "A Measurement-Based Stochastic Model for High-Speed Railway Channels". IEEE Transactions on Intelligent Transportation Systems, vol. 99, (2014), pp.1-16.

[2] B. Ai, X. Cheng and T. Kürner, "Challenges toward Wireless Communications for High-Speed Railway". IEEE Transactions on Intelligent Transportation Systems, vol.15, no.5, (2014), pp. 2143-2158.

[3] R. He, Z. Zhong, B. Ai and J. Ding, "An empirical path loss model and fading analysis for high-speed railway viaduct scenarios," IEEE Antennas Wireless Propagation, Letters, vol. 10, ( 2011), pp. 808-812.

[4] R. He, Z. Zhong, B. Ai, and J. Ding, "Propagation measurements and analysis for high-speed railway cutting scenario," Electronics Letters, vol. 47, no. 21, (2011), pp. 1167-1168.

[5] R. He, Z. Zhong, B. Ai, G. Wang, J. Ding and A. F. Molisch, "Measurements and analysis of propagation channels in high-speed railway viaducts," IEEE Transactions on. Wireless Communications., vol. 12, no. 2, (2013), pp. 794-805.

[6] R. He, Z. Zhong, B. Ai, J. Ding, Y. Yang and A. F. Molisch, "Short-term fading behavior in high-speed railway cutting scenario: measurements, analysis, and statistical models, " IEEE Transactions on Antennas and Propagation, vol. 61, no. 4, (2013), pp. 2209-2222.

[7] D. Jakominich, R. Krebs, D. Retzmann and A. Kumar, "Real time digital power system simulator design considerations and relay performance evaluation," IEEE Transactions on Power Delivery, vol. 14, no. 3, (1999), pp. 773-781.

[8] Z. Khalaf, A. Nafkha, and J. Palicot, "Blind spectrum detector for cognitive radio using compressed sensing and symmetry property of the second order cyclic autocorrelation, " in Proceedings of the 7th International ICST Conference on Cognitive Radio Oriented Wireless Networks and Communications 
(CROWNCOM), Stockholm, Sweden, (2012), pp. 291-296.

[9] Y. Zhang, Q. Shi and Y. Han, "Application of LabVIEW to Automated Measuring Dynamic Balancing of Motorcycle Crankshaft," in Proceedings of 2007 IEEE International Conference on Automation and Logistics, Jinan, China, (2007), pp. 3055-3059.

[10] S. Sen and B. Leung, "150 MHz 13b 12.5mW IF Digitizer using Sampling Mixer," in Proceedings of the Custom Integrated Circuits Conference, Santa Clara , USA, (1998), pp.233-236.

[11] M. Granieri, "Synthetic Instrumentation: An Emerging Technology," RF Design, vol. 27, no. 2, (2004), pp. 16-25.

[12] C. Ide,. B. Dusza, C. Wietfeld, "Performance of Channel-Aware M2M communications based on LTE network measurements," in Proceedings of IEEE International Symposium on Personal Indoor and Mobile Radio Communications (PIMRC), London, United kingdom, (2013), pp. $1614-1618$.

[13] X. Raimundo and S. Salous, "Bit error rate comparison of MATLAB multipath fading channel simulator and measurement based channel simulator using real measurement data," in Proceedings of 2012 International Symposium on Signals, Systems, and Electronics (ISSSE), Potsdam, Germany, (2012), pp. 1- 6 . 
International Journal of Future Generation Communication and Networking Vol. 8, No. 6 (2015) 